\title{
Categorical perception occurs in newly learned faces, other-race faces, and inverted faces
}

\author{
DANIEL T. LEVIN \\ Kent State University, Kent, Ohio \\ and \\ JAMES M. BEALE \\ Grand Valley State University, Allendale, Michigan
}

\begin{abstract}
On the basis of findings that categorical perception (CP) is possible in complex visual stimuli such as faces, the present study tested for CP on continua between unfamiliar face pairs. Results indicate that CP can be observed for unfamiliar faces, in both familiar (same-race) and unfamiliar (other-race) groups. In addition, significant CP effects were observed in inverted faces. Finally, half-continua were tested where midpoint stimuli became endpoints. This was done to ensure that stimulus artifacts did not account for the observed CP effects. Consistent with the perceptual rescaling associated with $\mathrm{CP}$, half-continua showed a rescaled CP effect. We argue that these CP effects are based on the rapid acquisition of perceptual equivalence classes.
\end{abstract}

Categorical perception $(\mathrm{CP})$ occurs when discriminations between stimuli within one category are more difficult than discriminations between stimuli that span the boundary between categories. The question is, under what conditions does this occur? Initial research suggested that $\mathrm{CP}$ would be observed only for naturally occurring continua and was perhaps a sign of innate or highly practiced predispositions to break these continua into equivalence classes (e.g., Liberman, Harris, Kinney, \& Lane, 1961). For example, Etcoff and Magee (1992) observed CP on continua between different facial expressions and suggested this supports the hypothesis that the perception of facial expression is innate. However, other findings suggest that such predispositions are not necessary to observe CP. In another study using faces, Beale and Keil (1995) found that participants perceive continua between individual familiar faces categorically. This is the case despite the likelihood that the continua between these faces have never been experienced by participants.

If $\mathrm{CP}$ is possible along artificial continua, then hypotheses assuming that $\mathrm{CP}$ is a sign of innate or even highly familiar perceptual categories are incorrect. Here, we explore two basic questions about the degree to which $\mathrm{CP}$ can be observed in the absence of previous learning. First, will it be possible to observe CP on novel continua between endpoints that do not have long-standing represen-

The authors thank Robert Goldstone and Bill Merriman for reading drafts of this report and Stevan Harnad and Andy Young for many helpful discussions. Correspondence should be addressed to D. T. Levin, Kent State University, Department of Psychology, Kent Hall, Kent, OH 44121-0001 (e-mail: dlevin@kent.edu). J. M. Beale can be reached at the Department of Psychology, Grand Valley State University, Allendale, MI 49401 (e-mail: bealej@river.it.gvsu.edu).

-Accepted by previous editor, Myron L. Braunstein tations associated with them as do familiar faces? A number of recent findings suggest that this kind of representation is not a necessary precondition for CP. This brings up the possibility that $\mathrm{CP}$ might be observed on continua between newly learned faces. Second, if CP occurs on novel continua between novel endpoints, then under what conditions will such effects be found? In this paper, we explored $\mathrm{CP}$ in newly learned faces and manipulated the degree to which face-specific coding processes were available by using faces from unfamiliar races and inverted faces.

\section{Categorical Perception: The Basic Phenomenon and Reasons to Expect It in Unfamiliar Faces}

The phenomenon of $\mathrm{CP}$ is interesting for a number of reasons, but perhaps the most basic is that $\mathrm{CP}$ involves cognitive and perceptual equivalence classes that affect our ability to perceive continuous variation among stimuli. In the basic CP paradigm, a set of stimuli are created that vary continuously along a single physical dimension. The simplest example is the color spectrum, which varies continuously in terms of wavelength. Despite continuity along the physical dimension of wavelength, we perceive a series of discrete color categories. Empirical verification of this impression usually comes from linked classification and discrimination tasks. The classification task, in which participants classify a set of stimuli using whatever labels are appropriate, determines the location of the boundaries between categories. For example, participants might be presented with a series of 11 hues ranging from $500 \mathrm{~nm}$ (red) to $600 \mathrm{~nm}$ (yellow) in $10-\mathrm{nm}$ steps and might be asked simply to label each "red" or "yellow." Label assignments can then be used to determine the boundary between red and yellow. Once this boundary is known, if these stimuli are perceived cate- 
gorically, one would predict more accurate discrimination between members of stimulus pairs that straddle the boundary between the colors, whereas discrimination between stimuli that lie within one of the regions will be less accurate (e.g., see Grahm, 1965). Facilitated discrimination accuracy between stimuli that straddle category boundaries implies that cognitive and perceptual processes act to divide physical stimulus continua into discrete equivalence classes. Differences among variants falling within a class are psychologically attenuated, whereas differences among variants that fall in a region between classes are amplified.

Initially, research on CP was confined to spoken language perception, where it appeared as though the continua between different phonemes were broken up by an innate language-specific process. For example, there exists a clear continuum between the phonemes $/ \mathrm{b} /$ and $/ \mathrm{p} /$ that can be defined in terms of the difference between the onset of voicing and the plosive burst. This difference is referred to as voice onset time (VOT). If voicing and the "burst" are simultaneous (VOT $=0$ ), the phoneme $/ \mathrm{b} /$ is perceived. If the VOT is greater than approximately $30 \mathrm{msec}$, the percept is $/ \mathrm{p} /$. It is therefore possible to create a continuum between these two phonemes by simply varying the VOT from 0 to $80 \mathrm{msec}$ in 10 -msec increments using a voice synthesizer. Each stimulus can then be paired with other stimuli on the continuum in a discrimination paradigm to test for differences in discriminability across the continuum. In the case of $/ \mathrm{b} /$ and $/ \mathrm{p} /$, pairs of stimuli that straddle the category boundary (for example, a 20-msec VOT vs. a 40-msec VOT) are easier to discriminate than pairs both falling to one side of the 30-msec boundary (Liberman, Cooper, Shankweiler, \& Studdert-Kennedy, 1967).

At first, CP was observed only with these stimuli, and not other language-like stimuli such as "inverted" synthetic phonemes (e.g., the high-frequency and lowfrequency formants were switched and their envelopes inverted; Liberman et al., 1961). This research was taken to support the hypothesis that $\mathrm{CP}$ was produced either by an innate perceptual screening mechanism particular to language that enhances the contrast between similar phonemes (Stevens, 1981) or by a perceptual inference based on the motor patterns required to make speech (Liberman et al., 1967). More recently, Etcoff and Magee (1992) argued that $\mathrm{CP}$ on continua between different facial expressions was similarly indicative of innate expression-specific perceptual mechanisms. For example, a continuum was created between a happy expression and a disgusted expression worn by the same individual. The presence of $\mathrm{CP}$ along this continuum was interpreted by the authors to imply that the particular facial expressions at each end of the continuum are specified innately. If one's theoretical preference is to avoid nativistic extrapolation, then the hypothesis that facial expressions are simply overlearned facial configurations also fits these findings. The key hypothesis in the above research is that $\mathrm{CP}$ results from specific long-standing representations that specify the continuum endpoints and, according to Etcoff and Magee, the actual continua themselves.

A number of experiments show, however, that $\mathrm{CP}$ does not necessarily imply innate programming, or even overlearning of the relevant stimuli. First, Kuhl (1981) found that chinchillas produced $\mathrm{CP}$ effects for the same phoneme continua that had been tested in humans, reproducing the exact VOT boundaries between different phoneme pairs as found in humans. This finding, in concert with others on infrahuman species (Kuhl \& Padden, 1982), cast doubt on the presumption that CP must necessarily reflect specific inborn phoneme boundaries. Kuhl (1987) instead suggests that low-level perceptual properties of the continua themselves contain naturally information-rich regions that the evolution of language has selected as dividing lines between phonemes.

In addition, the original findings that auditory $\mathrm{CP}$ was limited to speech sounds has been reversed. For example, Sawusch and Gagnon (1995) observed CP in nonspeech phoneme analogs. In this case, the critical manipulation that allowed CP was a task in which the entire stimulus continuum was played before alternate discrimination trials. Also, Burns and Ward (1978) found that music experts perceive musical intervals categorically. Smith, Kemler Nelson, and Appleton (1994) found that even musical novices could perceive continua between two-tone intervals categorically if each interval was associated with a well-known folk song (the subjects were informed that one tone interval was the first two notes of one song and the other represented the first two notes of another song). Thus, expertise allows CP to occur in novel unlabeled stimuli that mimic the practiced domain. Even where perceivers are less expert, if stimulus continua can be anchored by familiar endpoints, then CP is again observed.

The question is, how do Beale and Keil's (1995) findings fit with findings in music experts? They found CP on continua between familiar faces, but not unfamiliar faces. On the basis of this finding, they argue that CP is driven not by innate perceptual continua but rather by learned representations of the invariant structure of known faces. However, they reported no CP effects for unfamiliar faces, which appears to conflict with the above data on musical experts' perception of musical intervals. In the domain of music, expertise was associated with an ability to immediately represent new stimuli such that continua between them were perceived categorically. Novices were different in that they had to be provided with a direct mapping from the test stimulus to familiar music exemplars for CP effects to be found. In the domain of faces, it is safe to say that most adults are experts, but Beale and Keil's results make face experts similar to music novices in that neither showed $\mathrm{CP}$ effects on continua between novel exemplars.

Perhaps the conflict between the music expert data and Beale and Keil's (1995) face data can be resolved by specific aspects of their task. The experiment began with 
an instruction screen containing the names and images of the eight endpoint faces. Although four of the faces were well known and therefore probably required little learning, the other four were unfamiliar to most participants and therefore would have required at least some learning. Where they manipulated familiarity, Beale and Keil randomly mixed discrimination trials from continua between all eight faces within a single block. This intermixing might account for the lack of $\mathrm{CP}$ effects for the unfamiliar faces. If sufficient familiarity with novel faces must be built up during the discrimination trials, then Beale and Keil's task may not have allowed sufficient experience with each of the face continua to adequately represent all of the endpoints, especially for the novel stimuli. This possibility is supported by recent findings from Stevenage (1998), who had subjects complete a short-training task in which they learned to differentiate a pair of identical twins by classifying pictures of each with feedback. After the task, participants gave higher similarity ratings to different pictures of the same twin and lower ratings to pictures of different twins. The similarity ratings therefore suggested a CP effect based on short-term learning of previously unfamiliar faces. However, none of the above findings imply that long-term familiarity is unrelated to observing CP. Rather, the implication is that prior familiarity facilitates $\mathrm{CP}$ by eliminating the necessity that continuum endpoints be learned at some point during the experiment or on line during the actual task.

The present research therefore used a methodology similar to that of Beale and Keil (1995), with the exception that unfamiliar face pairs were presented from only one continuum in any given block of trials. If $\mathrm{CP}$ is possible with novel visual stimuli, then it may appear for unfamiliar faces if the learning task is made easier.

\section{Testing the Limits of $\mathrm{CP}$ in Faces}

If $\mathrm{CP}$ can be observed on novel continua and between novel endpoints, then it is important to ask about the extent of the phenomenon. It is possible that it will be observed on just about any continuum with sufficiently discriminable endpoints. Therefore, extensive expertise with a given stimulus domain may not be necessary to observe CP. For example, Goldstone (1994) found that it was possible to observe CP-like effects using continua of artificial visual stimuli given a small amount of classification training. The present experiments assessed the limits of CP in two ways. First, they included continua between faces from a less familiar race (Black faces for the White and Asian participants tested here), in addition to a more familiar race (White faces). Also included were continua that traverse the races. Given the well-known other-race recognition deficit (e.g., see Shapiro \& Penrod, 1986) whereby participants recognize faces from an unfamiliar race less well than those from a familiar race, it is straightforward to predict that no CP will be observed on continua between Black faces if the full compliment of face coding skills is a necessary precondition.
An additional test for the limits of $\mathrm{CP}$ was included in Experiment 2 in which all faces were presented upside down. This common manipulation is thought to differentially disrupt coding subtle variations in the configuration of facial features (Diamond \& Carey, 1986; Farah, Tanaka, \& Drain, 1995; Rhodes, Brake, \& Atkinson, 1993). This configural coding process is considered the hallmark of face coding and is probably the source of our ability to accurately differentiate members of this homogeneous class (Diamond \& Carey, 1986; Rhodes, Tan, Brake, \& Taylor, 1989). Therefore, if CP depends on expertise-based configural coding, any $\mathrm{CP}$ effects that appeared in Experiment 1 were expected to disappear in the inverted faces in Experiment 2.

\section{Summary and Predictions}

The goal of the present experiments was to improve our understanding of the $\mathrm{CP}$ phenomenon in complex visual stimuli such as faces by testing for $\mathrm{CP}$ on continua between unfamiliar faces. In Experiment 1, continua between pairs of faces were tested for the presence of CP. In this experiment, discrimination trials were presented in blocks including stimuli from a single continuum. In addition, continua within familiar and less familiar racial categories were included along with continua that spanned the two categories. In Experiment 2, the same stimulus set and procedures were used but the faces were inverted. This allowed a test for CP in the absence of expertiseinduced configural coding. Finally, Experiment 3 was designed to eliminate the possibility that stimulus artifacts were responsible for the positive findings in Experiments 1 and 2 .

\section{EXPERIMENT 1}

Experiment 1 was a standard CP paradigm based on the methodologies used in Etcoff and Magee (1992) and Beale and Keil (1995). Two linked tasks were used in this experiment: a discrimination task and a categorization task. The discrimination task was similar to one used by Beale and Keil in which participants viewed pairs of faces and were asked to respond which was more similar to one of the endpoint images. This task was used in place of the more traditional ABX paradigm primarily to focus participants' attention on the association between a given name and a face representation. In the categorization task, participants categorized each face used in the discrimination task according to their similarity to one or the other endpoint. This task therefore provided an estimate of the category boundary that was used to predict which pairs in the discrimination task should show improved performance. Pairs that straddle this boundary should show improved discrimination relative to the other face pairs.

In this experiment, and in all of the following experiments reported here, each participant completed discrimination tasks along three different continua: one with two Black faces as endpoints, one with two White endpoints, and one with one Black endpoint and one White end- 
point. Each continuum was presented in a different block of trials, and four different sets of three face pairs were used across different participants.

\section{Method}

Participants. A total of 17 participants completed Experiment 1. One participant who did not consistently classify the faces with one of the endpoints was dropped from the analysis. Of the 16 remaining participants, 1 was Asian and 15 were White. The participants completed the experiment in exchange for extra credit in a psychology course.

Stimuli. Sixteen unfamiliar male faces ( 8 Black and 8 White) were used as stimuli in this experiment. These were the same unfamiliar faces used in Levin (1996) and were equated for average distinctiveness (and rated familiarity) across the races. The 8 faces within each race were paired to make 4 continua within each race. In addition, 4 of the faces in each race were paired across race categories for a total of 12 face pairs. To equalize similarity among faces in each of the three groups (Black-Black, White-White, and Black-White continua), 10 judges rated the similarity of all possible pairings among the 16 faces. Judges were instructed to rate similarity based on the overall similarity of face shape using a 7-point scale $(1=$ very similar, $7=$ very dissimilar $)$ and were further instructed to ignore differences in race when viewing other-race pairs. On the basis of these ratings, sets of face pairs were chosen to equalize average similarity ratings between the three stimulus groups (mean similarity ratings: Black-Black pairs, 4.15 ; White-White pairs, 4.08; Black-White pairs, 4.05 ; $S D$ for all chosen pairs $=0.5823$ ).
Once the faces were paired, a set of 9 intermediate stimuli was created for each pair (using the morph program from Gryphon Software) by linearly interpolating the shape and luminance values on $10 \%$ intervals between the endpoint averages (see Beale \& Keil, 1995 , for details). Together with the continuum endpoints, the participants viewed 11 different stimuli representing controlled blends of the two faces (see Figure 1). As a matter of convention, $0 \%$ will be assigned to one end of the continuum and $100 \%$ to the other with intermediates described in terms of percentage locations on this scale. Therefore, the $50 \%$ stimulus is the intermediate that sits in the middle of the continuum. In the case of the Black-White (B-W) continua, the scale always begins with the Black face $(0 \%)$ and ends with the White face (100\%). On the White-White (W-W) and BlackBlack (B-B) continua, the $0 \%$ and $100 \%$ faces were arbitrarily chosen. However, as will become important later, the instruction screens containing the faces and names associated with them always had the $0 \%$ face on the left and the $100 \%$ face on the right.

Once the intermediates were created, all images were blurred slightly (0.5-pixel-radius Gaussian blur using Photoshop 2.0) to reduce the visibility of small image defects and interference patterns caused by scanning. Each image was approximately $240 \times 336$ pixels (horizontal $\times$ vertical) and was presented in 256-level gray scale.

Apparatus. The experiment was presented on Macintosh LC computers attached to 12 -in. color monitors set to 8-bit gray scale mode. Stimuli were viewed from approximately $60 \mathrm{~cm}$, and measured approximately $8.5 \times 12.5 \mathrm{~cm}$ on the monitor.

Procedure. The participants first completed three blocks of discrimination trials, one each with $\mathrm{B}-\mathrm{B}, \mathrm{W}-\mathrm{W}$, and $\mathrm{B}-\mathrm{W}$ continua. Before each block, the participants viewed an instruction screen
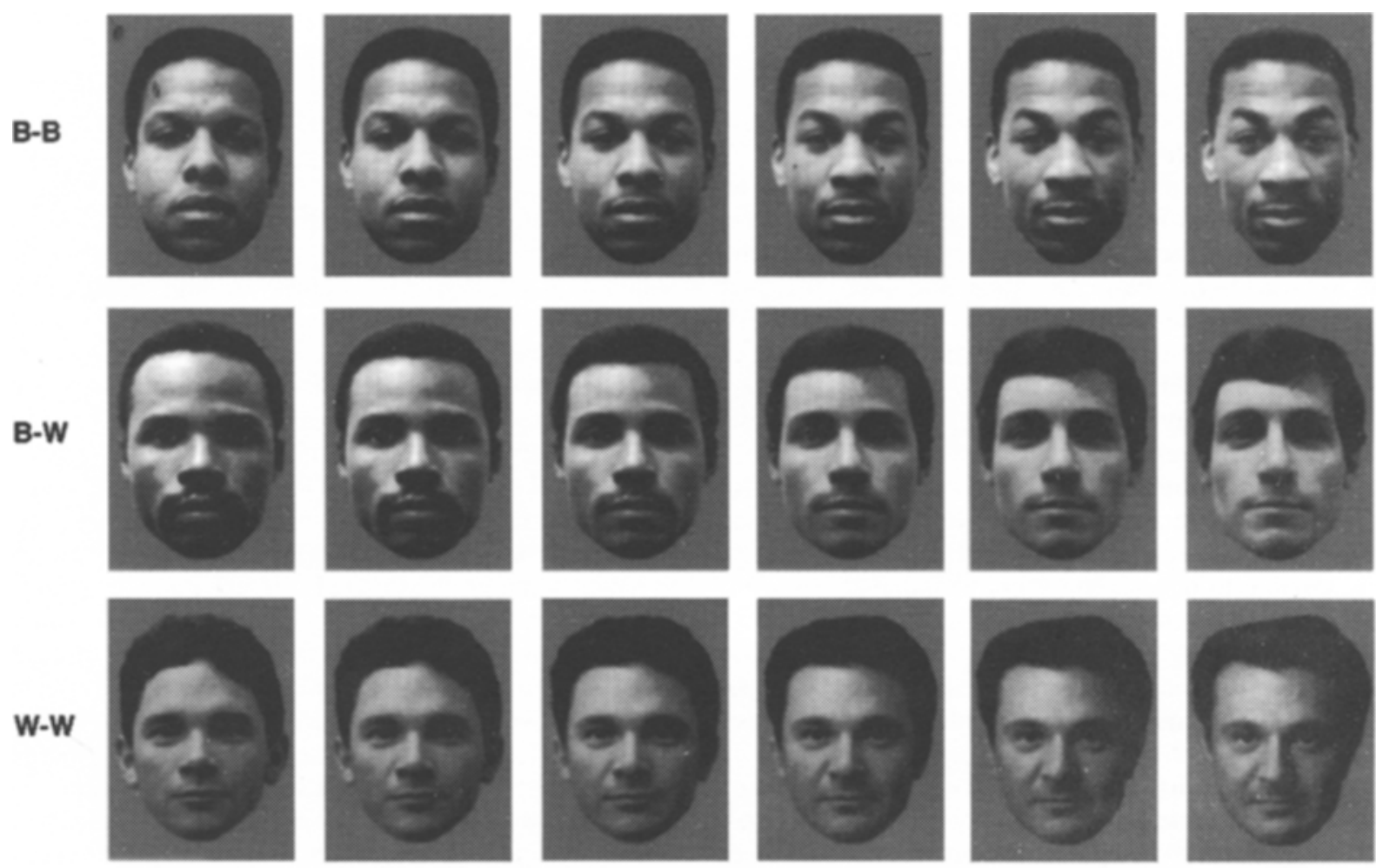

$0 \%$ End $100 \%$ Start

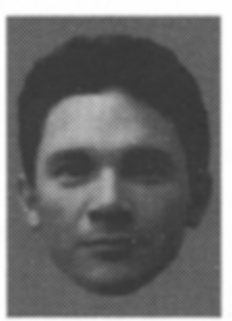

$20 \%$ End $80 \%$ Start

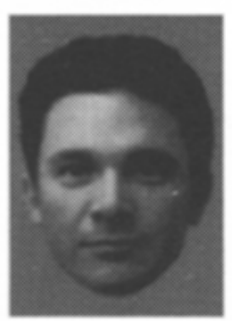

$40 \%$ End $60 \%$ Start

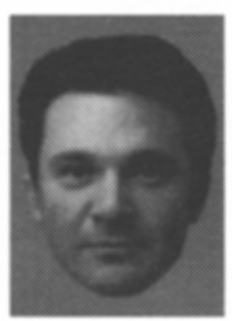

$60 \%$ End $40 \%$ Start

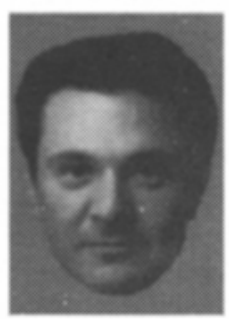

$80 \%$ End $20 \%$ Start

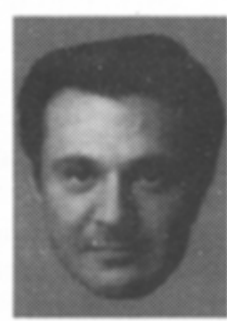

$100 \%$ End $0 \%$ Start

Figure 1. Sample set of stimuli. B-B continua have two Black faces as endpoints, B-W continua start at a Black face and end at a White one, and $W-W$ continua have two White faces as endpoints. Each type $(B-B, W-W$, and $B-W)$ was represented by four different continua with eight different faces as endpoints. 
that had the two endpoint faces, each associated with an arbitrarily chosen name (e.g., "Fred"). The participants were instructed to pay close attention to this screen and to be sure that they could identify each face and associate the correct name with it. On each trial of the discrimination task, the participants saw a response cue telling them to indicate which of the upcoming faces looked more like one of the endpoints. For example, if one of the endpoint faces was named "Fred," the participants might be asked, "Which of these faces looks more like Fred?" After reading the cue, the participants hit the space bar on the computer keyboard indicating their readiness to continue. They then saw two faces side by side for $1,000 \mathrm{msec}$. In all cases, the two faces in the stimulus display were separated by two steps, or $20 \%$ of the total continuum. After the faces disappeared, the participants responded by pressing the "l" key if the left face looked more like the target face or the " 2 " key if the right face did. In each block, there were 8 trials (two trials for each screen side and correct response) for each of the 9 face pairs along the continua for a total of 72 trials. The order of discrimination blocks was counterbalanced across participants such that half saw the other-race pair first and half saw one of the within-race pairs first and the other second. The order of the two within-race pairs was also counterbalanced.

After completing the discrimination trials, the participants completed three blocks of classification trials. The order of classification blocks followed the same order as the discrimination blocks for each participant. In each block, the participants classified individual faces along one of the continua from the discrimination trials. At the beginning of each block, the two endpoint faces were presented, again paired with their names. The participants were instructed to hit the " 1 " key if they thought an individual face was more similar to the first face or to hit the " 2 " key if it was more similar to the second face. Each of the 11 faces was presented three times for a total of 33 trials in each of the three blocks.

\section{Results}

For all analyses reported in this paper, data were collapsed across the four continua in each condition.

Classification task. The classification task allowed for selection of cross-boundary discrimination pairs using the same criterion as in Beale and Keil (1995) and Etcoff and Magee (1992). Pairs including one individual face classified with one of the endpoints on more than $66 \%$ of trials and another classified with the other endpoint on more than $66 \%$ of trials were selected as cross-boundary pairs to be compared with the other pairs that were considered within-category pairs.

By the above criteria, the $40 \%-60 \%$ pair was selected as the cross-boundary pair for the global analysis for all continuum types (B-B, W-W, and B-W; see Figure 2). This pair was also selected for individual analyses of the $\mathrm{B}-\mathrm{B}$ and $\mathrm{B}-\mathrm{W}$ continua. For the $\mathrm{W}-\mathrm{W}$ continua, both the $40 \%-60 \%$ and $50 \%-70 \%$ pairs straddled the $66 \%-$ $33 \%$ boundary, so both were selected to represent crossboundary discrimination accuracy (de Gelder, Teunisse, \& Benson, 1997).

Discrimination task. Data from the discrimination task were entered into one single-factor (AB Pair; $1 \%-$ $20 \%$ to $80 \%-100 \%$ ) analysis of variance (ANOVA) to test for $\mathrm{CP}$ across all continua in addition to three separate ANOVAs, one for each condition (B-B, B-W, W-W). To test for significant categorical perception effects, planned contrasts were computed between cross-boundary pairs and within category pairs to test (using one-tailed $t$ tests) for increased discrimination accuracy on the crossboundary pair.

The overall CP effect was highly significant $[F(1,15)=$ $\left.25.005, M S_{\mathrm{e}}=0.004, p=.0001\right]$. In addition, significant categorical effects were observed for all three continuum types (see Figure 2). For the B-W and W-W continua, cross-boundary pairs were more accurately discriminated than within-boundary pairs $[\mathrm{B}-\mathrm{W}, F(1,15)=14.775$, $M S_{\mathrm{e}}=0.008, p=.0008 ; \mathrm{W}-\mathrm{W}, F(1,15)=28.782, M S_{\mathrm{e}}=$ $0.011, p<.0001]$. The categorical effect was smaller but also significant for the $\mathrm{B}-\mathrm{B}$ condition $[F(1,15)=3.609$, $\left.M S_{\mathrm{e}}=0.015, p=.0385\right]$.

Average discrimination accuracy was $81.9 \%$ in the $\mathrm{B}-\mathrm{B}$ condition, $84.3 \%$ in the $\mathrm{B}-\mathrm{W}$ condition, and $82.3 \%$ in the $W-W$ condition. An ANOVA revealed no significant effect of continuum type on discrimination accuracy $(F<1)$.

Finally, as Figure 2 makes clear, discrimination accuracy was higher at the "beginning" of the continua than at the "end." Duncan tests indicate that discrimination accuracy was significantly lower for the $0 \%-20 \%$ pair than for the $80 \%-100 \%$ pair $(p<.05)$ and also lower for the $10 \%-30 \%$ pair than for the $70 \%-90 \%$ pair $(p<.01)$. A look at the individual continuum types shows that the $B-B$ and $W-W$ continua drive this effect. For the $B-B$ continua, accuracy was significantly lower for the $10 \%-$ $30 \%$ pair than for the $70 \%-90 \%$ pair $(p<.05)$; for the $\mathrm{W}-\mathrm{W}$ continua, accuracy was lower for both the $0 \%-$ $20 \%$ pair and the $10 \%-30 \%$ pair than for the $70 \%-90 \%$ and $80 \%-100 \%$ pairs, respectively $(p<.01)$. None of these comparisons was significant for the $\mathrm{B}-\mathrm{W}$ continua.

\section{Discussion}

In Experiment 1, face pairs on all continua showed a significant categorical effect, although the effect was smaller for the B-B pairs. These findings suggest that previous familiarity with continuum endpoints is unnecessary to observe CP. Instead, so long as participants get a chance to familiarize themselves with the stimuli, a sufficient representation is available that allows parsing of a visual continuum into equivalence classes.

One issue that arises when looking at Figure 2 is that no clear sharp peak in discrimination accuracy was evident for the $40 \%-60 \%$ stimulus pair. Instead, there appears to have been a gradual increase in accuracy as stimulus pairs approached the category boundary. Furthermore, discrimination accuracy was far above chance throughout the continuum; even the endpoint pairs were discriminated with $68 \%-75 \%$ accuracy. Both of these findings might be seen to conflict with "pure" CP whereby discrimination is only possible to the extent that stimulus pairs cross the category boundary. Therefore, discrimination accuracy within the perceptual categories should be at chance and should only increase above that level when one member of the pair falls into one category and the other member falls into the other category. This kind of finding is, however, not the norm, even in the case of phoneme discrimination (for review, see Pastore, 1987). 


\section{Classification Task}
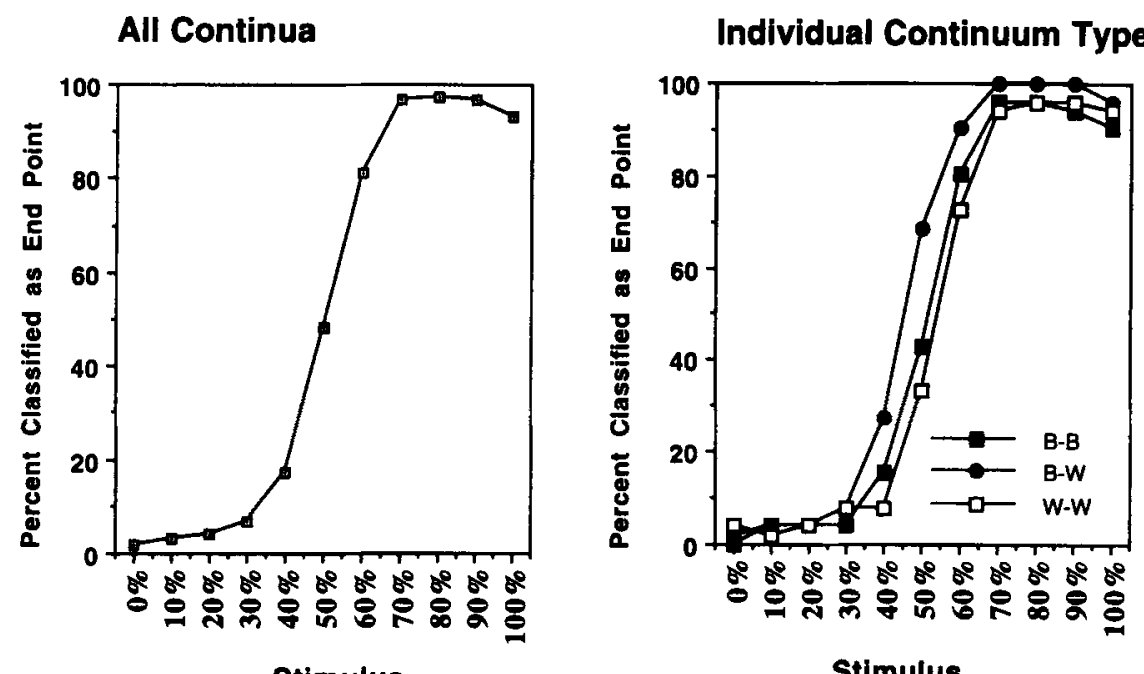

Stimulus

\section{Discrimination Task}

All Continua

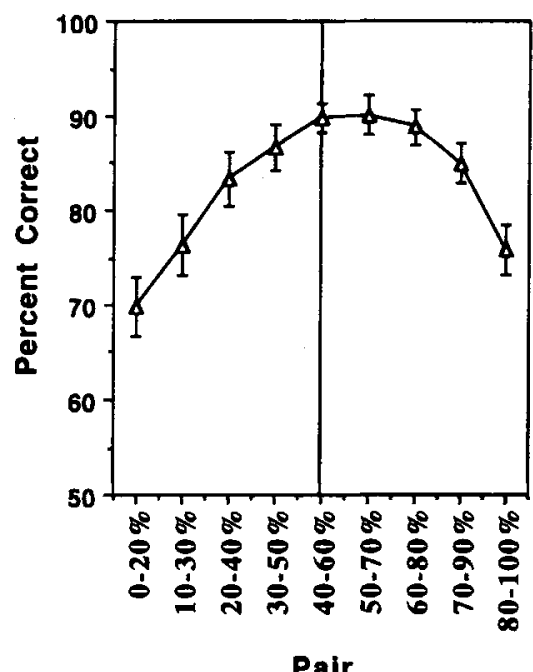

Individual Continuum Types

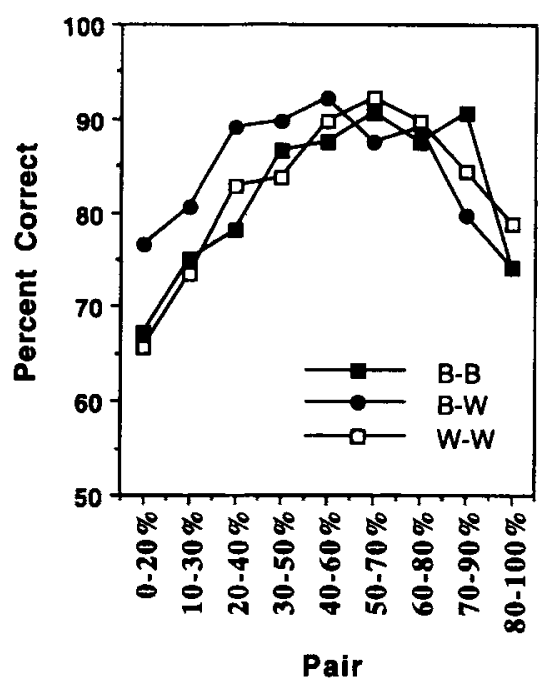

Figure 2. Classification and discrimination data from Experiment 1 . The $x$-axis of the classification graph represents the continua, including both endpoint stimuli $(0 \%$ and $100 \%)$ and intermediates $(10 \%-90 \%)$, and the $x$-axis of the discrimination graph represents stimulus pairs spanning the continua. The $0 \%$ and $100 \%$ endpoint designations are arbitrary for the $W-W$ and $B-B$ continua. For the $B-W$ continua, the $0 \%$ endpoint is the Black face of the pair, and the $100 \%$ endpoint is the White face. The classification graph contains the percentage of trials on which each stimulus was classified in the same category as the $100 \%$ (endpoint) stimulus. The discrimination graph contains discrimination accuracy for stimulus pairs along each continuum. The vertical line in the diserimination graph for all continua represents the pair crossing the category boundary.

Instead, discrimination accuracy is usually affected by some combination of factors. For example, Pisoni (1975) describes a model of $\mathrm{CP}$ that makes explicit the combined inputs of both categorical effects and perceptual effects (such as comparisons of iconic or echoic sensory traces) to determine discrimination accuracy throughout a con- tinuum. Therefore, above-chance discrimination within categories and unsharp peaks in accuracy are probably caused by noncategorical inputs to discrimination that are overlaid on the $\mathrm{CP}$ effect.

In this context, it is interesting to discuss the asymmetry evident in Figure 2 whereby discrimination accu- 
racy was higher at the "ends" of the continua than at their "beginnings." Although the between-race continua always began with the Black face and ended with the White face, the within-race continua were not intended to have any systematic difference between starting points and ending points. However, as noted in the Method section, the instruction screen in which each face pair was learned had the starting-point stimulus on the left side and the endpoint stimulus on the right. It is possible that this positioning in some way specified a relationship between the faces that caused increased discrimination accuracy for the right-side faces. One possibility is that the face on the left served as the "base" for comparison with the face on the left (Medin, Goldstone, \& Gentner, 1993). In the context of language, a sentence can specify a comparison between two concepts such that one, the "base," specifies the features relevant to the comparison, whereas the "target" is evaluated with reference to these features. If such a situation has occurred in the present case, the arrangement of the faces may have caused features of the face on the right to be coded relative to those on the left. This kind of selection could account for the increase in discrimination accuracy for variants of this face over that for the face on the left. While these possibilities are potentially interesting, a resolution of these issues will be reserved for future exploration and not further discussed here.

Before going on to Experiment 2, one potentially problematic aspect of the specific task used here needs to be addressed. On each trial, one of two stimuli must be matched with a representation of one endpoint face or the other. Therefore, on a subset of trials, the participants were asked which of two versions of one face looked like the other. For example, the participants might be asked which of the upcoming faces looks most like "John" and then be shown two faces that plainly look like "Fred." These trials might be particularly difficult because the specified target is a face at one end of the continuum, whereas the presented stimuli are from the other end. If so, then the observed $\mathrm{CP}$ effect might be based on reduced accuracy for these trials, especially because the problem would be particularly acute for stimulus pairs close to each endpoint. To eliminate this concern, the data were divided according to the relationship between the target specification and the discrimination stimuli. One analysis included only trials on which the target $(\mathrm{X})$ and discrimination (A and $B$ ) stimuli were from the same side ("near-X" trials) of the continuum, and the other included only trials for which the target was the endpoint opposite the discrimination stimuli ("far- $X$ " trials). If the task artifact caused the $\mathrm{CP}$ effect, then it should only be significant for the far- $X$ trials. However, this is plainly not the case. The CP effect is significant in both cases and is, in fact, stronger for the near-X trials [far-X, $F(1,15)=$ $12.016, M S_{\mathrm{e}}=0.005, p=.003$; near-X, $F(1,15)=25.137$, $\left.M S_{\mathrm{e}}=0.005, p=.0002\right]$. The implications of this particular pattern of results will be examined further in the General Discussion section.
The significant $\mathrm{CP}$ effect observed in other-race faces suggests that $\mathrm{CP}$ in unfamiliar faces generalizes to faces from unfamiliar groups. Given findings by Fallshore and Schooler (1995) and Rhodes et al. (1989) implying that configural coding is not present or reduced in other-race faces, it is possible that $\mathrm{CP}$ does not depend on the availability of this process. This runs counter to recent research suggesting that configural coding underlies the $\mathrm{CP}$ effect (Campbell, Pascalis, Coleman, Wallace, \& Benson, 1997; de Gelder et al., 1997). In both cases, CP was observed in upright faces and disappeared when the faces were inverted. This finding suggests the importance of configural coding because inversion has been found to substantially disrupt expertise-based coding of the relationships among features (Farah et al., 1995; Rhodes et al., 1993; Tanaka \& Farah, 1993).

Therefore, Experiment 2 tested the limits of $\mathrm{CP}$ by using inverted faces. If $\mathrm{CP}$ remained in inverted faces, then it is likely that configural coding was not necessary to observe this effect.

\section{EXPERIMENT 2}

\section{Method}

A total of 14 White participants completed Experiment 2 in exchange for course credit. Stimuli and procedures were identical to those in Experiment 1 with the exception that all faces (including the ones in the sample screens) were inverted by rotating them $180^{\circ}$.

\section{Results}

Classification task. Using the same criterion as in Experiment 1, the $40 \%-60 \%$ pair was again selected as the cross-boundary pair for all conditions (see Figure 3).

Discrimination task. The analysis was identical to that in Experiment 1. The test for $\mathrm{CP}$ across all continua was again highly significant $\left[F(1,13)=12.376, M S_{\mathrm{e}}=0.008\right.$, $p=.0019]$. Categorical effects were significant for the B-W continua $\left[F(1,13)=6.713, M S_{\mathrm{e}}=0.016, p=.0112\right.$ and the $\mathrm{W}-\mathrm{W}$ continua $\left[F(1,13)=4.125, M S_{\mathrm{e}}=0.026\right.$, $p=.0316]$ but nonsignificant for the $\mathrm{B}-\mathrm{B}$ continua $(F<1)$ (see Figure 3).

Average discrimination accuracy was $74.2 \%$ in the B-B condition, $78.3 \%$ in the $\mathrm{B}-\mathrm{W}$ condition, and $76.6 \%$ in the $\mathrm{W}-\mathrm{W}$ condition. None of the differences between conditions were significant, but discrimination accuracy was, overall, lower in Experiment 2 than in Experiment 1 [76.4\% vs. $\left.82.8 \% ; F(1,28)=5.497, M S_{\mathrm{e}}=0.017, p=.0264\right]$.

\section{Discussion}

Experiment 2 demonstrated $\mathrm{CP}$ in inverted faces, suggesting that configural coding processes are not a prerequisite to observe CP. As is evident from comparing Figures 2 and 3 , inversion seems to have reduced the size of the $\mathrm{CP}$ effect in that discrimination accuracy for endpoints was minimally reduced, whereas that for stimulus pairs in the middle of the continua was lower. Therefore, it seems reasonable to conclude that configural coding might 


\section{Classification Task}
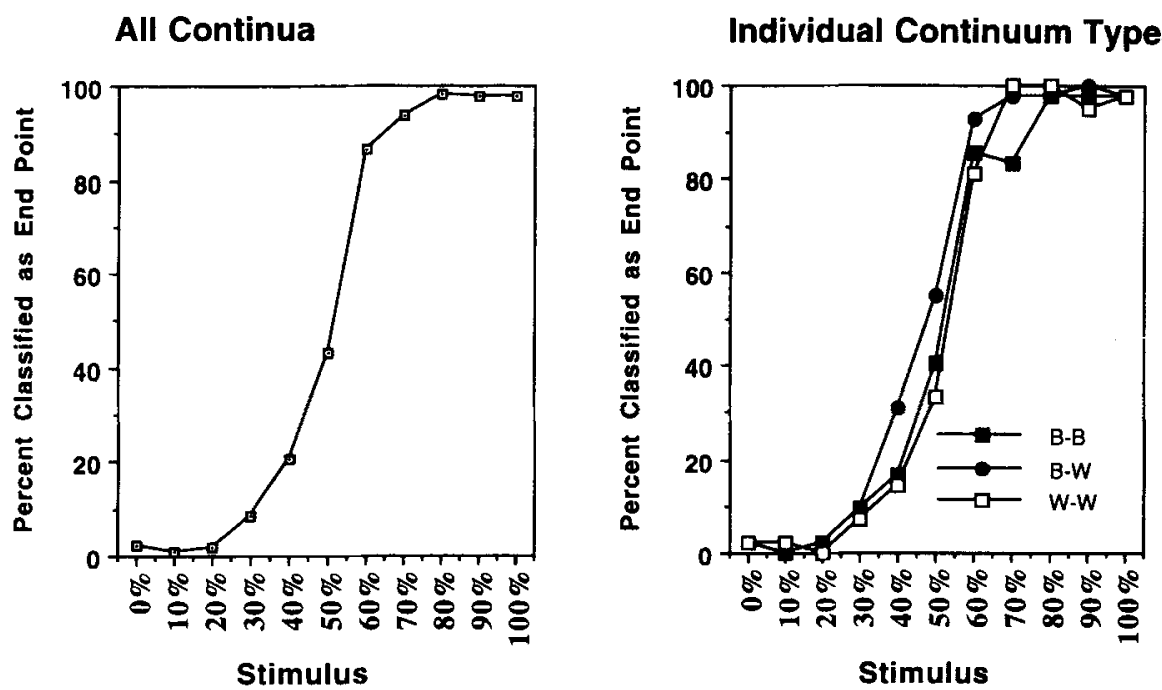

\section{Discrimination Task}

All Continua

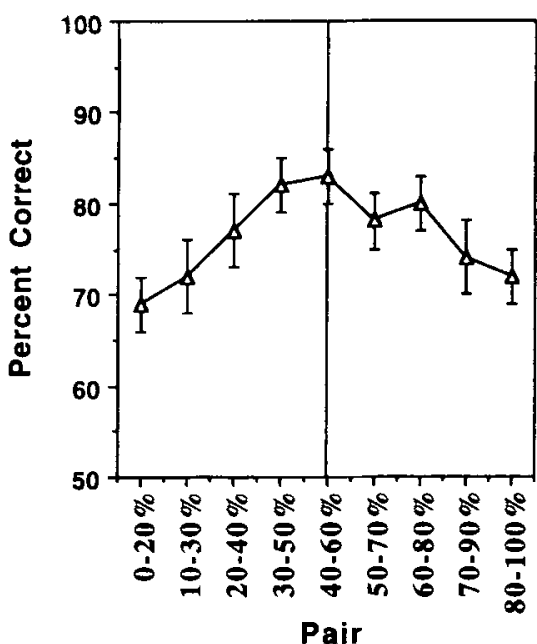

Individual Continuum Types

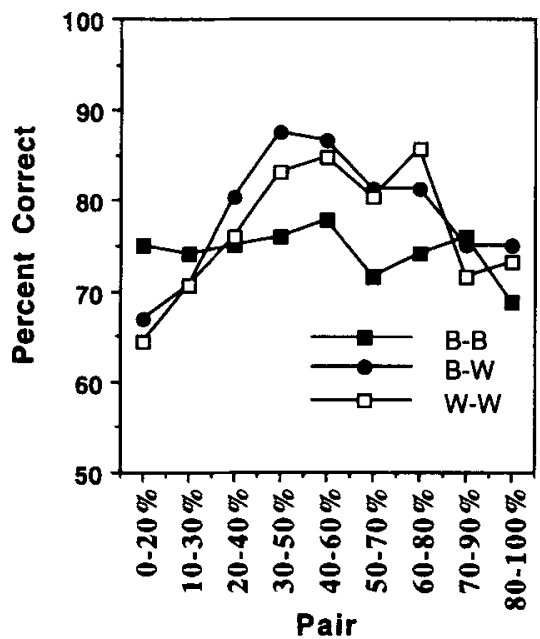

Figure 3. Classification and discrimination data from inverted faces used in Experiment 2. The $x$-axis of the classification graph represents the continua, including both endpoint stimuli $(0 \%$ and $100 \%)$ and intermediates $(10 \%-90 \%)$, and the $x$-axis of the discrimination graph represents stimulus pairs spanning the continua. The $0 \%$ and $100 \%$ endpoint designations are arbitrary for the $W-W$ and $B-B$ continua. For the $B-W$ continua, the $0 \%$ endpoint is the Black face of the pair, and the $100 \%$ endpoint is the White face. The classification graph contains the percentage of trials on which each stimulus was classified in the same category as the $100 \%$ (endpoint) stimulus. The discrimination graph contains discrimination accuracy for stimulus pairs along each continuum. The vertical line in the discrimination graph for all continua represents the pair crossing the category boundary.

increase the size of the $\mathrm{CP}$ effect, but it is not a prerequisite for observing it.

This finding suggests that $\mathrm{CP}$ in complex visual stimuli is not based on expertise-based configural coding and may instead be part of a much more general process. As reviewed in the introduction, it appears that $\mathrm{CP}$, or at least $\mathrm{CP}$-like phenomena, can be induced with novel vi- sual stimuli (Goldstone, 1994) and, in addition, can be observed in animals that lack long-term exposure to a given stimulus class (i.e., phonemes; Kuhl, 1981). These two findings are similar because they both imply that long-term experience, either with a stimulus class as a whole or with particular exemplars, is unnecessary to observe $\mathrm{CP}$. 


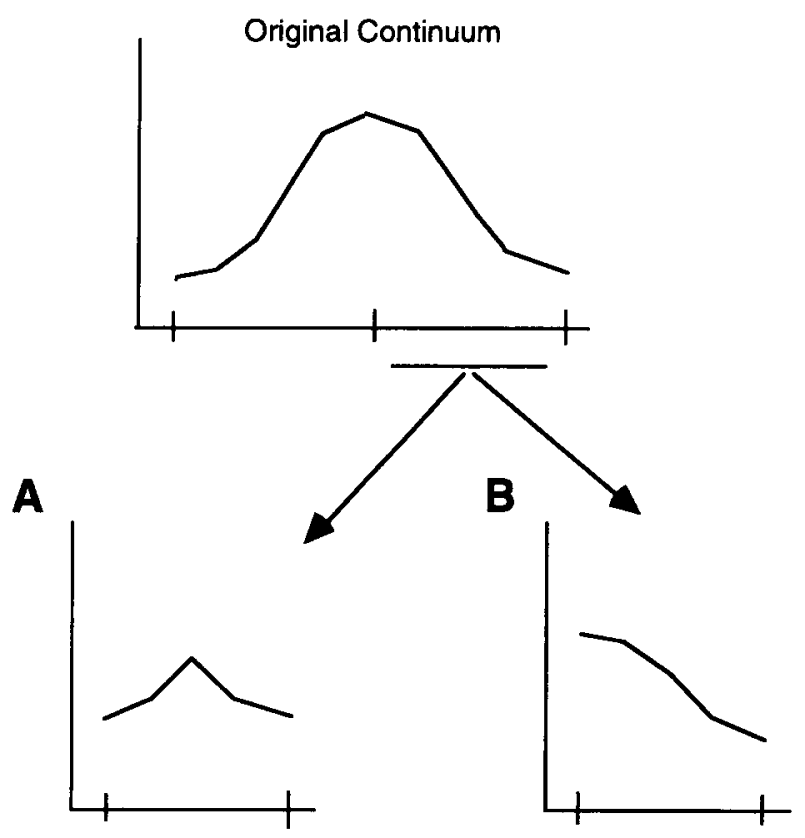

Categorical Perception

Figure 4. Predicted findings for half-continua assuming CP (A) or an artifact (B). If Experiments 1 and 2 revealed CP-like results because midregion stimuli contain artifacts that make them easy to discriminate, then redoing the experiment with half-continua (in which the stimulus originally serving as a midpoint is now an endpoint) should produce no CP effect. Instead, AB pairs that were originally in midregions should still be most accurately discriminated.

Although it is possible that $\mathrm{CP}$ can occur based on short-term learning, its presence in within-race continua and inverted faces does raise a number of concernsmost importantly, the possibility that the effect is a stimulus artifact. Whenever testing for CP between two complex visual stimuli, one must consider the possibility that the effect might be driven by accidental increases in the perceptual differences between midregion stimuli. For example, perhaps some aspect of the morphing process causes midregion stimuli to have a large number of small discontinuities or other artifacts that participants can use to enhance discrimination accuracy. In such a case, increased disciminability at category boundaries would be uninteresting. Although we attempted to reduce such concerns both by using Beale and Keil's (1995) "betterlikeness" task and by slightly blurring the stimuli, neither precaution completely eliminated the possibility that artifacts are driving our CP effects. Experiment 3 was therefore designed to provide a critical test of the hypothesis that artifacts are driving our findings.

\section{EXPERIMENT 3}

Experiment 3 was designed to test for artifacts by presenting participants with half-continua. In each of three blocks of trials, participants were shown face pairs that

were from half of a given continuum from Experiments 1 and 2 . Thus, one face was originally an endpoint, but the other was the midpoint of the original continua. If the categorical effect we have been observing is simply a stimulus artifact, accuracy should be high for stimulus pairs that originally straddled midpoints (which we will hereafter refer to as switch pairs) and low for all original endpoints. This is illustrated in Figure 4B, where accuracy on the half-continua simply mirrors that of the original continua. On the other hand, if the categorical effect results from the rapid acquisition of on-line representations of the endpoints, then a newly scaled CP effect should be observed where midregion stimuli that were originally the most accurately discriminated are now less accurately discriminated than the new midregion stimuli (see Figure 4). This hypothesis is clearest for the B-B and $\mathrm{W}-\mathrm{W}$ continuum types, for which the half-continua bear no relationship to specific categories. For the B-W half-continua, the switch pairs straddle the between-race boundary because they are always the middle of a continuum across race categories. Therefore, although the B-W may show rescaled CP effects, the switch pairs may well be accurately discriminated for nonartifact reasons.

Two separate discrimination conditions were run in Experiment 3: a "2-step" condition in which face pairs were separated by $33 \%$ of the half-continua (or $20 \%$ of the original continua and therefore identical in terms of physical difference to the stimulus pairs in Experiment 1), and a " 1 -step" condition in which face pairs were separated by $17 \%$ of the half-continua (or $10 \%$ of the original continua). In all cases, individual stimuli were presented upright and were identical to those used in Experiment 1.

\section{Method}

Participants. A total of 41 participants completed the discrimination and categorization tasks in exchange for course credit (22 in the 1 -step condition, and 19 in the 2-step condition). Of these, 6 were Asian, 1 was Indian, and 34 were White.

Stimuli. Stimuli were the same as those used in Experiments 1 and 2 with the exception that only half of each continuum was presented. Therefore, 2-step continua that previously consisted of nine face pairs, now consisted of only five pairs. The 1-step continua included six discrimination pairs. These pairs included stimuli ranging from $0 \%$ to $60 \%$ (or $40 \%$ to $100 \%$ ) of the original continua. This made it possible to use the discrimination pair that formerly straddled the midpoint at the continuum end. These pairs were the same as those used in Experiments 1 and 2, but, in the 2-step condition, they spanned $33 \%$ of the 7 -step continua instead of $20 \%$ of the 11 -step continua and, in the 1 -step continua, they covered $17 \%$ instead of $10 \%$ of the original continua. Two different versions of each continuum were created, each including one half or the other of the original continua. Each participant therefore viewed continua that represented one half or the other of the original continua, and no participant viewed two halves of the same original continuum.

Procedure. The procedure for the discrimination and categorization tasks was identical to that in Experiments $l$ and 2 with the exception that fewer trials were included in each block because of the reduced number of stimulus pairs. The instruction screens where names were paired with the faces were modified to include the former midpoint stimuli where appropriate. For each participant, both the discrimination and the categorization tasks used the same abbreviated continua. 
A Classification Task

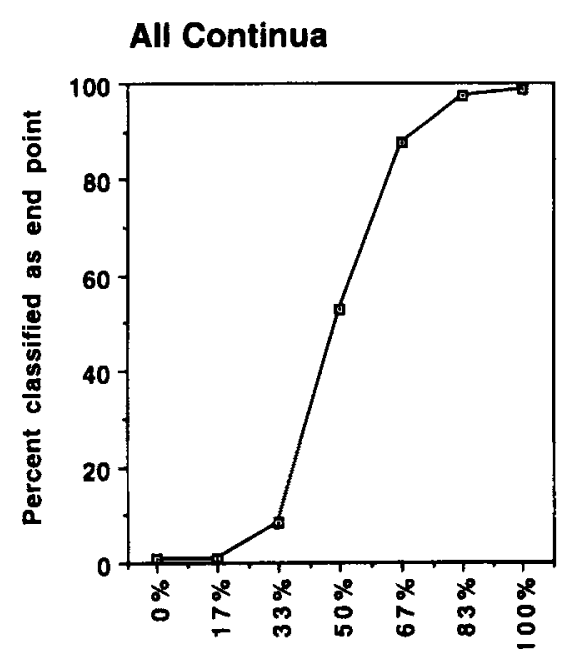

Stimulus
Individual Continuum Types

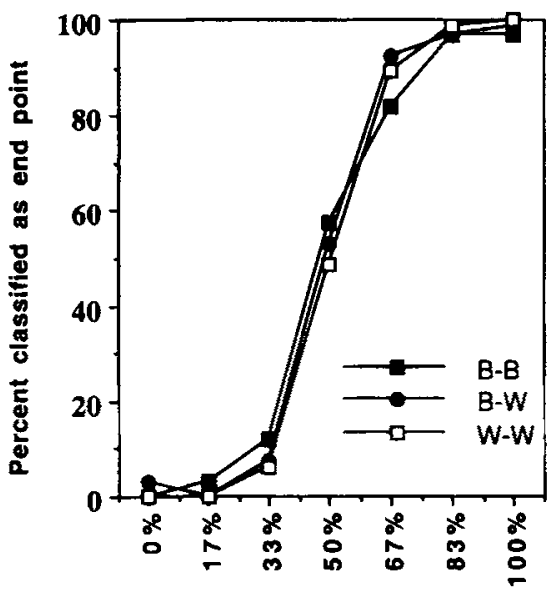

Stimulus

\section{Discrimination Task}

All Continua

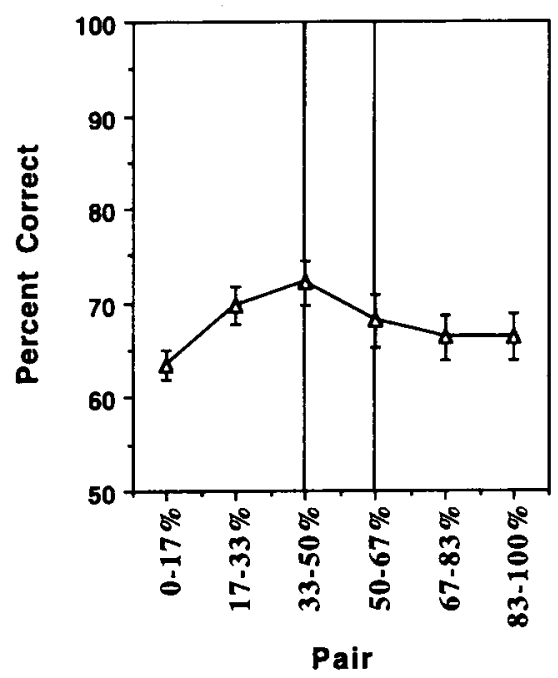

Individual Continuum Types

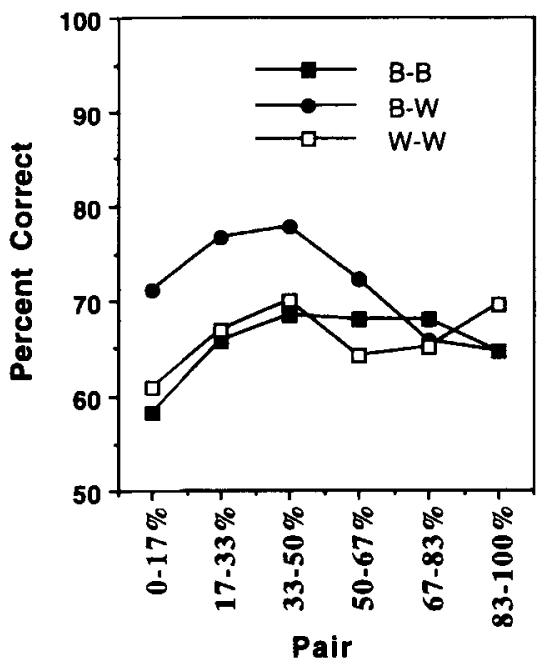

Figure 5A. Classification and discrimination data from the 1-step condition of Experiment 3 . The $x$-axis of the classification graph represents the continua, including both endpoint stimuli $(0 \%$ and $100 \%$ ) and intermediates $(10 \%-90 \%)$, and the $x$-axis of the discrimination graph represents stimulus pairs spanning the continua. The $0 \%$ and $100 \%$ endpoint designations are arbitrary for the $W-W$ and $B-B$ continua. For the $B-W$ continua, the $0 \%$ endpoint is the Black face of the pair, and the $100 \%$ endpoint is the White face. The classification graph contains the percentage of trials on which each stimulus was classified in the same category as the $100 \%$ (endpoint) stimulus. The discrimination graph contains discrimination accuracy for stimulus pairs along each continuum. The vertical lines in the discrimination graphs for all continua represent the pairs crossing the category boundary.

After completing the categorization and discrimination tasks, each participant was presented with an additional rating task. In the rating task, the participants saw each of the endpoint and midpoint faces used in the experiment (including faces from all four stimulus sets) and rated their distinctiveness on a 7-point scale, with anchor points ranging from very undistinctive (rating of 1 ) to very distinctive (rating of 7 ). The participants were instructed to rate the faces in terms of how well they would stand out in a crowd including male faces from many different races.

\section{Results}

As in Experiments 1 and 2, the pair or pairs straddling the physical middle of all continua (the $33 \%-67 \%$ in the 
B

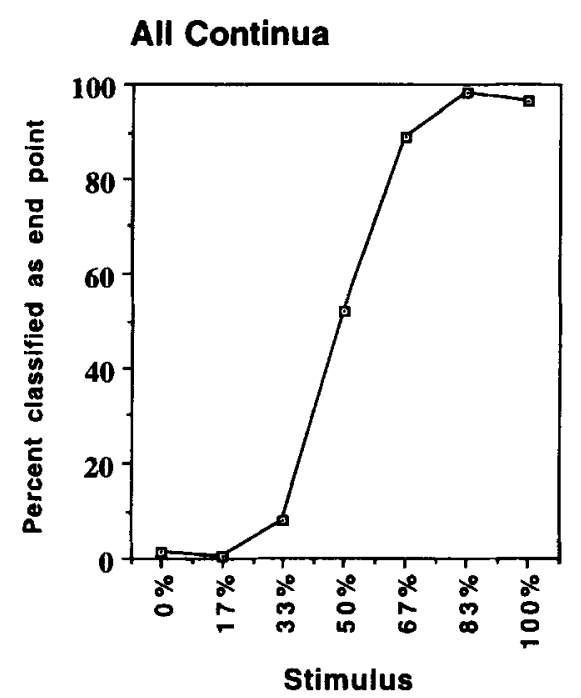

\section{Classification Task}

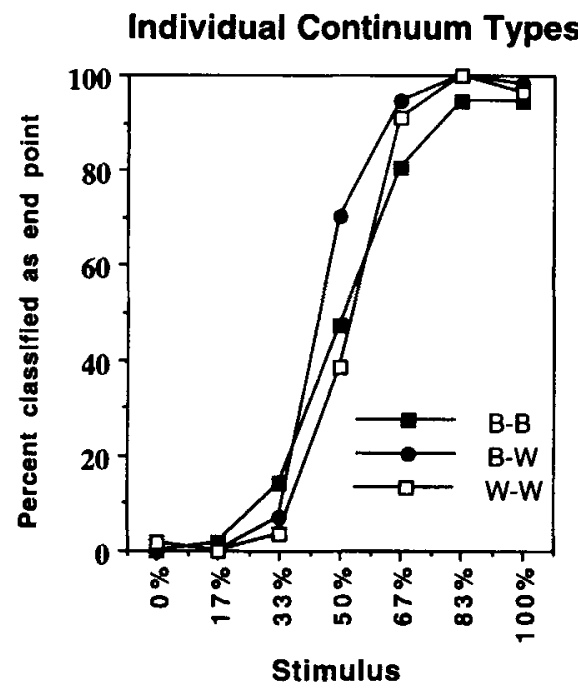

Discrimination Task

All Continua

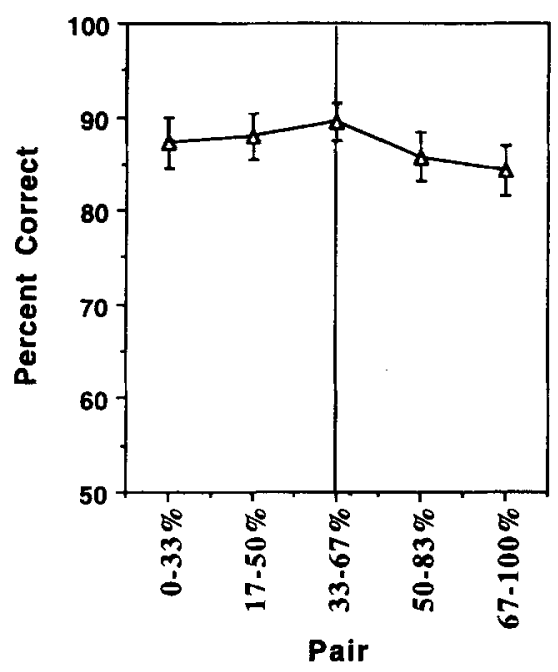

Individual Continuum Types

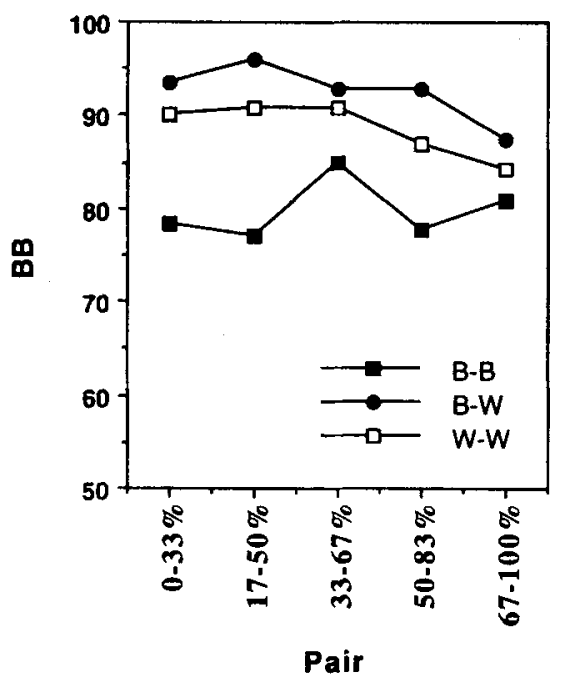

Figure 5B. Classification and discrimination data from the 2-step condition of Experiment 3. The $x$-axis of the classification graph represents the continua, including both endpoint stimuli $(0 \%$ and $100 \%)$ and intermediates $(10 \%-90 \%)$, and the $x$-axis of the discrimination graph represents stimulus pairs spanning the continua. The $0 \%$ and $100 \%$ endpoint designations are arbitrary for the $W-W$ and $B-B$ continua. For the $B-W$ continua, the $0 \%$ endpoint is the Black face of the pair, and the $100 \%$ endpoint is the White face. The classification graph contains the percentage of trials on which each stimulus was classified in the same category as the $100 \%$ (endpoint) stimulus. The discrimination graph contains discrimination accuracy for stimulus pairs along each continuum. The vertical lines in the discrimination graphs for all continua represent the pairs crossing the category boundary.

2 -step condition, and the $33 \%-50 \%$ and $50 \%-67 \%$ pairs in the 1 -step condition) also straddled the category bounds, as determined in the categorization tasks.

As can be seen in Figures 5A and 5B, small but significant $\mathrm{CP}$ effects were observed for both the 1-step and 2step conditions [1-step, $F(1,21)=4.763, M S_{\mathrm{e}}=0.009$, $p=.0203 ; 2$-step, $F(1,18)=7.361, M S_{\mathrm{e}}=0.002, p=$
$.0071]$. Overall tests including only the two within-race continuum types $(\mathrm{B}-\mathrm{B}+\mathrm{W}-\mathrm{W})$ were significant in the 2-step condition $\left[F(1,18)=14.93, M S_{\mathrm{e}}=0.002, p=\right.$ $.0006]$ and marginally significant in the 1 -step condition $\left[F(1,21)=2.576, M S_{\mathrm{e}}=0.013, p=.0617\right]$. In the $1-$ step condition, a significant categorical effect was observed for the B-B continua $\left[F(1,21)=4.382, M S_{\mathrm{e}}=\right.$ 
$0.013, p=.0243]$, whereas the effect was nonsignificant for the $\mathrm{W}-\mathrm{W}$ continua $(F<1)$, and marginally significant for the B-W continua $\left[F(1,21)=2.391, M S_{\mathrm{e}}=0.029\right.$, $p=.0685]$. In the 2 -step condition, the CP contrasts for the $\mathrm{B}-\mathrm{W}$ and $\mathrm{W}-\mathrm{W}$ continua were nonsignificant, although, for the $\mathrm{W}-\mathrm{W}$ continua, they came close $[\mathrm{B}-\mathrm{W}$, $\left.F<1 ; \mathrm{W}-\mathrm{W}, F(1,18)=2.365, M S_{\mathrm{e}}=0.005, p=.0775\right]$. The CP contrast for the $\mathrm{B}-\mathrm{B}$ continua was, however, significant $\left[F(1,18)=4.664, M S_{\mathrm{e}}=0.013, p=.0223\right]$.

Planned contrasts were also run to test for increased discrimination accuracy for stimuli that had been in the midregions of the original continua (as predicted by an artifact hypothesis). For the 1 -step condition, this involved a contrast between accuracies for the $0 \%-17 \%+$ $17 \%-33 \%$ pairs and the $67 \%-83 \%+83 \%-100 \%$ pairs (recall that the former pairs are switch pairs and the latter are the continuum end). For the 2-step condition, the contrast was computed between the $0 \%-33 \%+17 \%-$ $50 \%$ pairs and the $50 \%-83 \%+67 \%-100 \%$ pairs. Onetailed tests were used in all cases. These contrasts were nonsignificant for the overall accuracies in both conditions [1-step, $F<1 ; 2$-step, $F(1,18)=1.979, M S_{\mathrm{e}}=0.007$, $p=.0883]$. Within the 1 -step condition, the contrast did not approach significance for the $\mathrm{B}-\mathrm{B}$ and $\mathrm{W}-\mathrm{W}$ continua $(F \mathrm{~s}<1)$. The test was significant for the B-W continua $\left[F(1,21)=5.701, M S_{\mathrm{e}}=0.032, p=.0132\right]$. Within the 2-step condition, the contrast was significant for the W-W continua $\left[F(1,18)=3.375, M S_{\mathrm{e}}=0.014, p=.0414\right]$ and nonsignificant for the $\mathrm{B}-\mathrm{W}$ and $\mathrm{B}-\mathrm{B}$ conditions [B-W, $\left.F(1,18)=2.520, M S_{\mathrm{e}}=0.016, p=.0649 ; \mathrm{B}-\mathrm{B}, F<1\right]$. Thus, in the overall tests and in three of the four critical individual tests (e.g., the B-B and W-W tests for 1-step and 2-step conditions; recall that existing race categories could cause switch pairs to be accurately discriminated in the B-W continua), there was no evidence of an artifact contribution to discrimination accuracy.

In addition to testing for $\mathrm{CP}$ using contrasts between cross-boundary pairs and within-category pairs, we tested for significant linear and nonlinear trends using polynomial regression. This allowed a test of nonlinearities, as predicted by $\mathrm{CP}$, independent of possible linear trends, as predicted by stimulus artifacts. In all cases, the independent variable was pair number ( 1 for the switch pair, and 5 or 6 for the original endpoint pair), and the dependent variable was percent accuracy for that pair across all continuum types. For the 1-step condition, a third-order regression was the most parsimonious model $\left(r^{2}=.963\right.$; fourth order, $r^{2}=.966$; second order, $\left.r^{2}=.605\right)$ and was associated with a near-significant overall $F$ test $[F(3,5)=$ $17.5, p=.0545] . \mathrm{X}, \mathrm{X}^{2}$, and $\mathrm{X}^{3}$ terms were all associated with significant beta coefficients $(B \mathrm{~s}=.221,-.062$, and $.005 ; p \mathrm{~s}=.0281, .0363$, and .0475 , respectively). Thus, as predicted by a CP finding, there were significant nonlinearities in the data. In addition, the significant linear component was associated with a positive beta coefficient (increased accuracy for pairs near the original endpoint), goes in a direction opposite to that predicted by an artifact hypothesis (increased accuracy for the switch pair). None of the regressions (linear, second-order, and third order) run on the data from the 2-step condition approached overall significance, so they were not analyzed further. ${ }^{1}$

Another interesting pattern was evident in the 2-step condition. Note that, in this condition, the physical difference between stimuli within each pair was identical to that in Experiment 1. As compared with Experiment 1, however, overall discrimination accuracy significantly increased for stimulus pairs on the B-W continua [Experiment $1,84.3 \%$; Experiment $3,92.5 \% ; F(1,33)=8.265$, $\left.M S_{\mathrm{e}}=0.007, p=.007\right]$ and also increased for the W-W continua, although the effect was only marginally significant [Experiment 1, 82.3\%; Experiment 3, 88.6\%; $\left.F(1,26)=3.825, M S_{\mathrm{e}}=0.009, p=.059\right]$. Accuracy remained unchanged for the B-B continua (Experiment 1 , $81.9 \%$; Experiment 3, 79.7\%; $F<1$ ).

\section{Discussion}

Experiment 3 provides evidence that the CP effects observed in Experiments 1 and 2 were not driven by stimulus artifacts. In both the 1-step condition and the 2-step condition, the overall CP effect was again significant. Switch pairs that formerly straddled the category boundary and therefore were most accurately discriminated in Experiments 1 and 2, now included the new endpoint and, in many cases, were least accurately discriminated. On the half-continua, the CP effect was rescaled relative to that in Experiments 1 and 2, which demonstrates its dependence on the particular location of each discrimination pair relative to the continuum endpoints. If the effects were artifacts, based on low-level perceptual differences between the stimuli along the original continua, then discrimination accuracy should reflect each pair's place on these continua and be unaffected by its location on the new continua.

Although new CP effects were observed, a small asymmetry was observed for one of the individual continuum types in which switch pairs were more accurately discriminated than the corresponding end pairs for one of the four tests on critical continuum types (the W-W 2step condition). Switch pairs were also somewhat more accurately discriminated in the 1-step and 2-step B-W continua, but, as mentioned above, this can be expected because the switch pairs straddle the between-race category boundary even if they are defined as endpoints in the context of the experiment. Thus, it appears that the location of stimulus pairs on the original continua may have a small effect on discrimination accuracy. Although this effect might reflect the contribution of a stimulus artifact, there is another plausible explanation. This alternative begins with the assumption that intermediates are generally less distinctive than endpoints because they are essentially averages of the endpoint face.

To confirm this, we asked 11 new participants to rate the midpoint and endpoint faces for distinctiveness and found that midpoint faces were less distinctive (rating of 3.46 on a scale of $1-7)$ than endpoint faces $[4.29 ; F(1,10)=$ 41.431, $\left.M S_{\mathrm{e}}=0.277, p=.0001\right]$, a finding confirmed 
recently by Busey (1998). Given that distinctive faces are more accurately recognized than typical ones (Going \& Read, 1974; Light, Kayla-Stuart, \& Hollander, 1979; Valentine \& Bruce, 1986), it is likely that they are associated with stronger or more robust representations. If this is true, then discrimination accuracy might have been reduced in continuum end regions because of a "perceptual magnet" effect (Iverson \& Kuhl, 1995; Kuhl, 1991) whereby strong representations of a stimulus cause near variants to be assimilated into the stimulus class and therefore are inaccurately discriminated. It is possible that such an effect contributed to the discrimination accuracies observed here. The key is that different locations on simple continua between faces vary in terms of distinctiveness, and this variation could contribute to categorical effects. Thus, the tendency for switch pairs to have a slight advantage in discrimination could be an artifact, but it could also reflect a more interesting variant of Kuhl's perceptual magnet effect. However, it is important to note that on no half-continua were the former midpoints discriminated most accurately. This clearly suggests that location on the newly defined continua was a strong determinant of discrimination accuracy.

In addition to the data from Experiment 3, other findings mitigate against an artifact explanation. First, Levin (1999) used the same stimulus set in a design where stimuli from three different continua were intermixed in a single block of trials. In this case, no CP effect was observed. In addition, the negative finding in unfamiliar faces reported by Beale and Keil (1995) was based on different stimuli but the same distortion process. Therefore, both of these findings converge to suggest the implausibility of a simple artifact explanation. If the $\mathrm{CP}$ effects we have observed here were based on a stimulus artifact induced by the distortion process, then the effect should not disappear when mixing of different continua makes the learning task more difficult. Finally, the $\mathrm{CP}$ effect size varied systematically with the difficulty of representing the continuum endpoints. In Experiment 1, the effect was weaker for other-race faces (same-race, $F=28.782$; otherrace, $F=3.609$ ). Inversion in Experiment 2 also weakened the effect in same-race faces $(F=4.125)$, while completely eliminating it in other-race faces $(F<1)$. Again, these results would not be expected if the effect were a simple perceptual artifact. Instead, the CP finding appears linked to the relative difficulty that participants have in representing the continuum endpoints.

One surprising finding from the 2-step condition is that overall discrimination accuracy on the $\mathrm{B}-\mathrm{W}$ and $\mathrm{W}-\mathrm{W}$ continua improved for the same-face pairs when they were part of half-continua, as compared with full continua. Thus, when the continua included at least one face from the familiar group, discrimination accuracy seems to be heavily influenced by the percentage of the continuum spanned by each pair as opposed to the actual physical difference between pairs. The lack of between-experiment improvement on B-B continua did not occur, because the participants spent little time looking at B-B end- points, as compared with $\mathrm{B}-\mathrm{W}$ and $\mathrm{W}-\mathrm{W}$ endpoints. The mean inspection time for $\mathrm{B}-\mathrm{B}$ instruction screens was actually slightly greater than that for $\mathrm{B}-\mathrm{W}$ and $\mathrm{W}-\mathrm{W}$ continua (B-B, $41.9 \mathrm{sec}, S E=5.2 \mathrm{sec}$; B-W: $38.9 \mathrm{sec}$, $S E=3.9 \mathrm{sec} ; \mathrm{W}-\mathrm{W}, 36.1 \mathrm{sec}, S E=5.6 \mathrm{sec}) .{ }^{2}$ It therefore appears likely that improved discrimination accuracy on $\mathrm{B}-\mathrm{W}$ and $\mathrm{W}-\mathrm{W}$ continua was the result of rescaling the perceptual difference between face pairs based on the psychological difference between the endpoints. This process is similar to the range effects observed when making discriminations between different tone intensities and pitches (Gravetter \& Lockhead, 1973; Lockhead \& Hinson, 1986). For example, Lockhead and Hinson (1986) found that intensity discrimination between two tones is more accurate if the task includes a third tone that is similar to the first two than if the third is very different. Thus, the perceptual difference between the two tones is effectively greater if they are part of a larger set that covers a small intensity range.

\section{GENERAL DISCUSSION}

Two main findings emerge from these data. First, $\mathrm{CP}$ is possible on continua between novel faces. Second, CP is observed even when the faces are inverted and when the faces belong to an unfamiliar group. It therefore appears that $\mathrm{CP}$ can be observed in a wide variety of situations. Given this, it appears that a good understanding of CP must take into account the on-line learning processes that allow us to set up visual representations of individual objects.

\section{Learning New Perceptual Categories}

In the research reported here, there were two primary opportunities for the participants to learn the faces. First, for each of three blocks of trials, they viewed an instruction screen containing the two endpoint faces, each associated with an arbitrarily chosen name. As reported in Experiment 3, it appears that the participants spent an average of approximately $40 \mathrm{sec}$ viewing this screen. Given that these averages were based on only the second and third blocks of trials, it is probably accurate to assume that this time was spent primarily on the faces and not on reading the instructions that were simply repeats of those on the first screen. Most of this time was probably spent closely comparing the faces with each other and attempting to associate a name with each. Moreover, verbal instructions given to the participants emphasized the importance of getting the face-name association correct and further emphasized that this task would take some thought and was not as easy as it appeared. These instructions were given to avoid the tendency to quickly look at the stimuli and then continue the task, which could result in forgetting the correct face-name association, a necessary precondition for making responses.

The second major source of learning occurs during the discrimination trials themselves. During this task, participants are gradually exposed to the continuum and must assess the degree to which the intermediates look more 
or less like the endpoints. Again, the task context could play a central role in determining the extent of the $\mathrm{CP}$ effect. In addition, it is important to ask about the time course of this learning: Will the CP effect be observed in the first blocks of discrimination trials, or does it only appear late in the experiment? An exploratory analysis of the data from Experiment 1 shows that the CP effect is present even if only the first half of the discrimination trials are analyzed $\left[F(1,15)=10.219, M S_{\mathrm{e}}=0.011, p=\right.$ $.0060]$. Therefore, it appears that $\mathrm{CP}$ reflects a quick parse of new perceptual categories into equivalence classes.

If the $\mathrm{CP}$ we have observed arises during the experiment itself, then what specific processes cause it? One means of approaching the question is to ask whether the effect is caused by reduced sensitivity to variation within perceptual categories ("equalization"), thereby causing less accurate discrimination in continuum end regions, or by increased sensitivity to variation on the borders of perceptual categories, thereby causing more accurate discrimination in continuum midregions ("sensitization"; Goldstone, 1994; Pisoni, 1991). In the present case, there is no direct evidence on the matter, but a comparison between Experiments 1 and 2 does give some hints. Inverting the faces seems to have reduced the $\mathrm{CP}$ effect primarily by reducing discrimination accuracy in the continuum midregions. Discrimination accuracy for the $0 \%-20 \%$ and $80 \%-100 \%$ stimulus pairs was relatively unaffected by inversion (upright, $72.8 \%$; inverted, $70.5 \%$ ), whereas accuracy for the other more central pairs was more clearly reduced [upright, $85.7 \%$; inverted, $78.0 \%$; orientation $X$ region (end $/ \mathrm{mid}$ ) interaction, $F(1,28)=4.127, M S_{\mathrm{e}}=$ $0.003, p=.0518]$. It therefore appears that inverting the faces reduced the $\mathrm{CP}$ effect primarily by reducing discrimination accuracy in the midregion, implying that the effect is based on sensitization more than equalization.

This conclusion is similar to that arrived at by Goldstone (1994), who tested the effects of categorization training on the degree to which discrimination accuracy could be affected for only part of a given dimension. Those experiments included a training task in which classifications were based on dimensions, such as the size and luminance of gray squares, such that two values fell into each of two categories. Goldstone found that training increased discrimination accuracy selectively for values that straddled the trained category boundaries and found no evidence that it reduced accuracy for values within a category.

In contrast to Goldstone (1994), Livingston, Andrews, and Harnad (1998) recently observed learned CP effects characterized by equalization within categories. Similar to in Goldstone's study, subjects were exposed to a learning task in which they classified stimuli on the continuum into two categories and then completed a discrimination task. Compared with subjects who had not done the learning task, subjects who learned the categories showed a CP effect characterized by equalization within categories. As Livingston et al. point out, a number of factors may have produced the contrast between their data and Goldstone's. The most important is that they used a similarity-judging task in which CP was indicated by judgments of increased similarity within categories and decreased similarity between categories. Goldstone used a same/different task in which subjects who learned the categories were more accurate for between-category pairs. Livingston et al. discuss two task-relevant factors that may have produced this difference. First, their similarityrating task allowed for continuous responses (a rating ranging from 1 to 9), whereas Goldstone's task involved binary responses. Furthermore, Goldstone's task involved presentation of stimulus pairs that were the same, whereas Livingston et al.'s did not. Our data give tentative support to the former hypothesis-we also used a task with a binary response to reveal sensitization but did not present pairs of identical stimuli. Another contrast between Livingston et al.'s, and Goldstone's and our data is that the latter tasks were performance-based and the former were subjective ratings. Perhaps the similarity ratings depended more on decision-stage processing, whereas the performance tasks depended on more immediate perceptual processes. In any case, the reasons behind this empirical contrast remain open to debate and could certainly prove the basis for fruitful exploration.

In addition to suggesting a putative sensitization effect, our data go a step further and show that $\mathrm{CP}$ can be observed for novel continua, even in the absence of pretraining. In particular, the sensitization we observed was not the result of a training task where each member of the sensitized discrimination pairs were actually classified into different categories. Instead, we observed sensitization based more directly on representations of the continuum endpoints. No experience classifying one member of a pair in category A and the other in category B was necessary for the cross-boundary pair to be discriminated more accurately. This implies that the learned effects we have observed are based less on sensitization produced by direct training and more on the way that representations of the endpoints themselves cause increased sensitivity to variation in intermediates. The comparison between the data reported here and the data reported by Beale and Keil (1995) reinforces this conclusion. As mentioned above, Beale and Keil mixed different continua in a single block of discrimination trials and found no CP on continua between unfamiliar faces. Thus, increasing the number of faces that must be represented decreases the CP effect.

In suggesting the importance of endpoint representations, these data run counter to adaptation level theory as a sole explanation for the $\mathrm{CP}$ effects we have observed here. According to this explanation, $\mathrm{CP}$ is caused by a low-level adaptation process in which the mean value of the stimulus dimension becomes an adapted "neutral point" (Helson, 1948). During the discrimination task, stimuli are judged relative to the point. Pairs that straddle the adapted region are therefore easy to discriminate, and pairs that fall within one side of the adaptation level are more difficult to discriminate (see Wilson, 1987, for review). Thus, the range of physical stimulus values comprising the continuum cause $\mathrm{CP}$, not representations of 
the endpoint per se. The close correspondence of CP effect size with codability of the endpoints suggests that something more than repetition-based adaptation caused the CP effects we have observed here.

However, it might be possible to modify an adaptation level explanation of CP to encompass our results. For example, it would be helpful to add some mechanism by which neutral points become less robust in difficult-tocode faces or when a number of different continua are presented in a single block of trials. In the latter case, adaptation might overlap between continua to produce diffuse neutral points that affect discrimination on more than one continuum. This could reduce the $\mathrm{CP}$, especially on continua between unfamiliar faces that vary between previously unknown values.

The near-pair and far-pair comparison in Experiment 1 also suggests the incompleteness of a range-based explanation for these CP effects. It is possible that midregion stimuli were more accurately discriminated because they are effectively judged within a smaller range than end-region stimuli because no stimulus is highly different from them. According to research discussed above, a given physical difference between stimuli might become psychologically smaller if they are coded as part of a large range. These effects can occur both globally and locally (Repp \& Libermann, 1987). In the latter case, the trials immediately preceding the stimuli to be discriminated have a particularly strong effect on the effective range. Although range effects do appear to contribute to overall discrimination accuracy (see discussion of Experiment 3), the near- $X /$ far- $X$ analysis in Experiment 1 reveals a stronger $\mathrm{CP}$ effect when the stimulus pair was on the same side of the continuum as the target stimulus, a circumstance in which the effective stimulus range is reduced. A range theory appears to predict a smaller $\mathrm{CP}$ effect here if one assumes that the $\mathrm{X}$ stimulus is effective in defining a range locally. Therefore, the relationship between the representation invoked on each trial and the perceived stimuli mediated the effect - a circumstance outside the scope of most range-based theories, which refer more directly to the effects of repeatedly viewing the stimuli themselves.

\section{Summary and Conclusions}

These findings suggest that the scope of the CP phenomenon should be expanded from the idea that it represents innate perceptual constraints, or constraints brought on only by long-term familiarity with the particular stimuli used in the experimental setting. Instead, it might be helpful to view CP as a dynamic on-line process that parses perceptual input in response to both long-term needs and task-specific constraints (Goldstone, 1994). This kind of on-line classification is similar to that proposed by Barsalou (1993) for general-knowledge concepts and therefore highlights the similarities in form between research on abstract concepts and perceptual classes discussed by Medin and Barsalou (1987). As a practical matter, we should expect to observe CP in many situations where participants have the opportunity to sufficiently differentiate continuum endpoints either during training trials or when simply shown them. As Harnad (1987) points out, this process might exemplify the global similarity between psychophysical identification and naming in that both processes are based on the need to quickly assimilate the extent of equivalence classes. Although CP is facilitated by expertise with the category to which the stimuli belong, this skill may not be strictly necessary. As such, the presence of $C P$ per se tells us little about whether continua or specific stimulus classes are innately specified or are even highly learned. Instead, CP can serve as a useful index of short-term perceptual learning, which can help us understand the first steps in molding perceptual variation into cognitively useful equivalence classes.

\section{REFERENCES}

Barsalou, L. W. (1993). Flexibility, structure, and linguistic vagary in concepts: Manifestations of a compositional system of perceptual symbols. In A. F. Collins, S. E. Gathercole, M. A. Conway, \& P. E. Morris (Eds.), Theories of memory (pp. 29-101). Hove, England: Erlbaum.

Beale, J. M., \& KeIL, F. C. (1995). Categorical effects in the perception of faces. Cognition, 57, 217-239.

Burns, E. M., \& WARD, W. D. (1978). Categorical perceptionphenomenon or epiphenomenon: Evidence from experiments in the perception of melodic musical intervals. Journal of the Acoustical Society of America, $\mathbf{6 3}, 456-468$.

BusEy, T. A. (1998). Physical and psychological representations of faces: Evidence from morphing. Psychological Science, 9, 476-483.

Calder, A. J., Young, A. W., Perrett, D. I., Etcoff, N. L., \& RowLAND, D. (1996). Categorical perception of morphed facial expression. Visual Cognition, 3, 81-117.

Campbell, R., Pascalis, O., Coleman, M., Wallace. S. B., \& BenSON, P. J. (1997). Are faces of different species perceived categorically by human observers? Proceedings of the Roval Society of London: Series $B, \mathbf{2 6 4}, 1429-1434$.

de Gelder, B., Teunisse, J., \& Benson, P. J. (1997). Categorical perception of facial expressions: Categories and their internal structure. Cognition \& Emotion, 11, 1-23.

Diamond, R., \& Carey, S. (1986). Why faces are and are not special: An effect of expertise. Journal of Experimental Psychology: General, 115, 107-117.

Etcoff, N. L., \& Magee, J. J. (1992). Categorical perception of facial expression. Cognition, 44, 227-240.

FallShORE, M. F., \& SCHOOL.ER, J. W. (1995). The verbal vulnerability of perceptual expertise. Journal of Experimental Psychology: Learning, Memory, \& Cognition, 21, 1608-1623.

Farah, M. J., Tanaka, J. W., \& Drain, H. M. (1995). What causes the face inversion effect? Journal of Experimental Psychology: Human Perception \& Performance, 21, 628-634.

GoING, M., \& READ, J. D. (1974). Effects of uniqueness, sex of subject, and sex of photograph on facial recognition. Perceptual \& Motor Skills, 39, 109-110.

GoLDSTONE, R. (1994). Influences of categorization on perceptual discrimination. Journal of Experimental Psychology: General, 123, 178-200.

Grahm, C. H. (1965). Discriminations that depend on wavelength. In C. H. Grahm (Ed.), Vision and visual perception (pp. 452-503). New York: Wiley.

Gravetter, F., \& Lockheed. G. R. (1973). Criterial range as a frame of reference for stimulus judgement. Psychological Review, 80, 203-216.

HARNAD, S. (1987). Category induction and representation. In S. Harnad (Ed.), Categorical perception: The groundwork of cognition (pp. 535-565). New York: Cambridge University Press. 
Helson, H. (1948). Adaptation level as a basis for a quantitative theory of frames of reference. Psychological Review, 55, 297-313.

IVERSON, P., \& KUHL, P. K. (1995). Mapping the perceptual magnet effect for speech using signal detection theory and multidimensional scaling. Journal of the Acoustical Society of America, 97, 553-562.

KUHL, P. K. (1981). Discrimination of speech by nonhuman animals: Basic auditory sensitivities conducive to the perception of speechsound categories. Journal of the Acoustical Society of America, 70, 340-349.

KUHL, P. K. (1987). The special-mechanisms debate in speech research: Categorization tests on infants and animals. In S. K. Harnad (Ed.), Categorical perception: The groundwork of cognition (pp. 355-386). New York: Cambridge University Press.

KUHL, P. K. (1991). Human adults and human infants show a "perceptual magnet effect" for the prototypes of speech categories, monkeys do not. Perception \& Psychophysics, 50, 93-107.

KuHL, P. K., \& PADDEN, D. M. (1982). Enhanced discriminability at the phonetic boundaries for the voicing feature in macaques. Perception \& Psychophysics, 32, 542-550.

LEVIN, D. T. (1996). Classifying faces by race: The structure of face categories. Journal of Experimental Psychology: Learning, Memory, \& Cognition, 22, 1364-1382.

LEVIN, D. T. (1999). Race as a visual feature: Using visual search and perceptual discrimination tasks to understand face categories. Manuscript submitted for publication.

Liberman, A. M., Cooper, F. S., Shankweiler, D. P., \& StuddertKennedy, M. (1967). Perception of the speech code. Psychological Review, 74, 431-461.

Liberman, A. M., Harris, K. S., Hoffman, H. S., \& Griffith, B. C. (1957). The discrimination of speech sounds within and across phoneme boundaries. Journal of Experimental Psychology, 54, 358-368.

Liberman, A. M., Harris, K. S., KinNey, J., \& Lane, H. (1961). The discrimination of relative onset-time of the components of certain speech and nonspeech patterns. Journal of Experimental Psychology, 61, 379-388

Light, L. L., Kayla-Stuart, F.. \& Hollander, S. (1979). Recognition memory for typical and unusual faces. Journal of Experimental Psychology: Human Learning \& Memory, 5, 212-228.

Livingston, K. R., Andrews, J. K., \& Harnad, S. (1998). Categorical perception effects induced by category learning. Journal of Experimental Psychology: Learning, Memory \& Cognition, 24, 732-753.

LockHEAD, G. R., \& Hinson, J. ( 1986). Range and sequence effects in judgment. Perception \& Psychophysics, 40, 53-61.

MEdin, D. L., \& BarSalou, L. W. (1987). Categorization processes and categorical perception. In S. Harnad (Ed.), Categorical perception: The groundwork of cognition (pp. 455-490). New York: Cambridge University Press.

Medin, D. L., Goldstone, R. L., \& Gentner, D. (1993). Respects for similarity. Psychological Review, 100, 254-278.

PAStORE, R. E. (1987). Categorical perception: Some psychophysical models. In S. Harnad (Ed.), Categorical perception: The groundwork of cognition (pp. 535-565). New York: Cambridge University Press.

PISONI, D. B. (1975). Auditory short-term memory and vowel perception. Memory \& Cognition, 3, 7-18.

PISONI, D. B. (1991). Modes of processing speech and nonspeech signals. In I. G. Mattingly \& M. Stewart-Kennedy (Eds.), Modularity and the motor theory of speech perception (pp. 225-238). Hillsdale, NJ: Erlbaum.

RePP, B. H., \& Libermann, A. M. (1987). Phonetic category boundaries are flexible. In S. Harnad (Ed.), Categorical perception: The groundwork of cognition (pp. 535-565). New York: Cambridge University Press.

Rhodes, G., Brake, S., \& Atkinson, A. P. (1993). What's lost in inverted faces? Cognition, 47, 25-57.

Rhodes, G., Tan, S., Brake, S., \& TAylor, K. (1989). Expertise and configural encoding in face recognition. British Journal of Psychology, 80, 273-283.
Sawusch, J. R., \& GaGnON, D. A. (1995). Auditory coding, cues, and coherence in phonetic perception. Journal of Experimental Psychology: Human Perception \& Performance, 21, 635-652.

Shapiro, P. N., \& PENrod, S. (1986). Meta-analysis of facial identification studies. Psychological Bulletin, 100, 139-156.

Smith, J. D., Kemler Nelson, D. G., \& Appleton, T. (1994). What child is this? What interval was that? Familiar tunes and music perception in novice listeners. Cognition, 52, 23-54.

SteVenaGe, S. V. (1998). Which twin are you? A demonstration of induced categorical perception of identical twin faces. British Journal of Psychology, 89, 39-57.

STEVENS, K. N. (1981). Constraints imposed by the auditory system on properties used to classify speech sounds: Data from phonology, acoustics and psycho-acoustics. In T. F. Meyers, J. Laver, \& J. Anderson (Eds.), The cognitive representation of speech (pp. 61-74). Amsterdam: North-Holland.

TAnaka, J. W., \& Farah, M. J. (1993). Parts and wholes in face recognition. Quarterly Journal of Experimental Psychology, 46A, 225-245.

Valentine, T., \& BRUCE, V. (1986). Recognizing familiar faces: The role of distinctiveness and familiarity. Canadian Journal of Psychology, 40, 300-305.

WiLSON, M. (1987). Brain mechanisms in categorical perception. In S. Harnad (Ed.), Categorical perception: The groundwork of cognition (pp. 387-417). New York: Cambridge University Press.

\section{NOTES}

1. To allay the concern that the statistical test for CP used for Experiments 1-3 was too liberal, we reanalyzed the data using another traditional test for CP (e.g., Liberman, Harris, Hoffman, \& Griffith, 1957). In this kind of test, the categorization results are used to predict discrimination accuracy for each pair. Following Calder, Young, Perrett, Etcoff, and Rowland (1996), we started with a baseline estimate for within-category accuracy by averaging accuracy for the first and last pairs in the continuum. Added to this was the absolute difference between the A and B stimuli in probability of classification with a continuum end multiplied by .25 to scale estimates in rough accord with the overall accuracy data. Regressions tested the relationship between predicted and observed discrimination accuracies in each condition. For Experiment 1, increased accuracy in continuum ends was considered a noncategorical input to discrimination accuracy and was accounted for by adding a "side" variable to a multiple regression. Accounting for continuum side, the relationship between predicted and actual accuracy was significant $(p<.05)$ overall and for all individual continuum types (partial $r \mathrm{~s}=.74, .78, .77$, and .82 , for $\mathrm{B}-\mathrm{B}, \mathrm{B}-\mathrm{W}, \mathrm{W}-\mathrm{W}$, and overall average continua, respectively). In Experiment 2, a simple regression (there was no side effect in this experiment) predicted discrimination scores significantly for $\mathrm{B}-\mathrm{W}, \mathrm{W}-\mathrm{W}$, and overall average continua $(r \mathrm{~s}=.80$, .68 , and .79 , respectively), but not for B-B continua $(r=.43)$. Data from the two conditions in Experiment 3 were combined to allow increased power (which was lacking because there were only 5 or 6 pairs in each condition), and a multiple regression including variables for predicted accuracy and condition was run. Controlling for condition, predicted accuracy was significant overall (partial $r=.68$ ) but not for individual continuum types (partial $r \mathrm{~s}=.50, .50$, and .30 , for $\mathrm{B}-\mathrm{B}, \mathrm{B}-\mathrm{W}$, and $\mathrm{W}-\mathrm{W}$ continuum types, respectively).

2. These means include only the second two of three instruction screen inspection times for each participant. The first instruction screen for each participant was present on each computer before the participant sat down to begin the task; therefore, the "RT" from that stimulus was not a measure of the time spent actually looking at it. Thus, means across conditions reflected contributions from different sets of participants, and, therefore, no test of significance is reported.

(Manuscript received October 13, 1997; revision accepted for publication December 13, 1998.) 